\title{
IDENTIFICACIÓN MORFOLÓGICA Y MOLECULAR DE ESPECIES AUTÓCTONAS TRICHODERMA SPP., AISLADAS DE SUELOS DE IMPORTANCIA AGRÍCOLA
}

\section{MORPHOLOGICAL AND MOLECULAR IDENTIFICATION OF NATIVE TRICHODERMA SPP. ISOLATED FROM SOILS OF AGRICULTURAL IMPORTANCE}

\author{
María Delfina Sánchez Miranda ${ }^{1}$ \\ Luis Francisco Moreno Mayorga ${ }^{2}$ \\ Leandro Alberto Páramo Aguilera *3
}

(recibido/received: 30-marzo-2021; aceptado/accepted:17-mayo-2021)

RESUMEN: Trichoderma sp. es un género de hongo que está siendo utilizado ampliamente como una alternativa sostenible para el control de enfermedades de plantas y promotor de crecimiento en cultivos de importancia agrícola. Tiene una gran diversidad genética, por lo que el presente estudio pretende identificar y describir morfológica y molecularmente especies provenientes de diferentes fuentes tan diversas como suelo agrícola, tomate triturado, cultivo de plátano, pastos entre otros. En el estudio se encontró 17 aislados de hongos con características en sus colonias similares a Trichoderma spp., los cuales después de observar sus características morfológicas se identificaron presuntivamente como pertenecientes a este género. La caracterización de su conidióforo, fiálides y conidias, demostró que dichos aislados correspondían al género Trichoderma spp. Posteriormente se demostró que los 17 aislados pertenecían al género Trichoderma sp mediante secuenciación, utilizando los cebadores ITS1 (5 TCC GTA GGT GAA CCT GCG G 3) ADNr, encontrándose 7 especies diferentes del hongo en el estudio, las cuales fueron: T. harzianum, T. viride, $T$. asperellum, $T$. asperelloide, $T$. songyi, $T$. virens y $T$. breve. De estas especies $T$. asperellum y $T$. harzianum, son de vital importancia por ser previamente identificadas como efectivos agentes de control biológico de enfermedades y promotores de crecimiento de plantas y se abre una nueva puerta de investigación con T. breve y T. songyi,

\footnotetext{
${ }^{1}$ Universidad Nacional Autónoma de Nicaragua-León. Departamento de Agroecología. Escuela de Ciencias Agrarias y Veterinaria. León Nicaragua.

${ }^{2}$ Universidad Nacional Autónoma de Nicaragua-León. Departamento de Agroecología. Escuela de Ciencias Agrarias y Veterinaria. León Nicaragua.

${ }^{3}$ Universidad Nacional de Ingeniería. Programa de Investigación, Estudios Nacionales y Servicios del Ambiente (PIENSA). Managua, Nicaragua. *leandro.paramo@piensa.uni.edu.ni; 1paramo2014@gmail.com ,Autor para correspondencia.
} 
ya que muy poco se conoce sobre la aplicación biotecnológica de éstas.

PALABRAS CLAVE: Identificación molecular, Trichodermas, Promotor de crecimiento, análisis filogenético, hongos antagonistas.

ABSTRACT: Trichoderma sp. is a genus of fungus that is being used widely as a sustainable alternative for the control of plant diseases and growth promoter in crops of agricultural importance. It has a great genetic diversity Therefore, this study aims to identify and describe morphologically and molecularly species from different sources as diverse as agricultural soil, crushed tomato, plantain cultivation, pastures, among others. In the study, 17 fungal isolates were found with characteristics in their colonies similar to Trichoderma spp. Which after observing their morphological characteristics were presumptively identified as belonging to this genus. The characterization of its conidiophore, phialides and conidia, showed that these isolates corresponded to the genus Trichoderma spp. Subsequently, it was demonstrated that, the 17 isolates belonged to the genus Trichoderma sp by sequencing, using the ITS1 primers (5 TCC GTA GGT GAA CCT GCG G 3) rDNA, finding 7 different species of the fungus in the study, which were: $T$. harzianum, T. viride, T. asperellum, T. asperelloide, T. songyi, T. virens and $T$. brief. From these species, $T$. asperellum and $T$. harzianum, are of vital importance because they have been previously identified as effective agents for the biological control of diseases and plant growth promoters and a new door of research with $\mathrm{T}$. breve and $\mathrm{T}$. songyi, is opened since very little is known about the biotechnological application of these.

KEYWORDS: Molecular identification, Trichodermas, Growth promoter, Phylogenetic analysis, Antagonistic fungus.

\section{INTRODUCCIÓN}

El género Trichoderma representa a nivel mundial una amplia variedad de organismos con una alta relevancia económica y ambiental, debido a su potencial como agentes de control biológico de organismos fitopatógenos que afectan cultivos de importancia agrícola y su capacidad de promover el crecimiento de las plantas (Martínez, et al., 2013). Muchas de las especies de Trichoderma han demostrado, según estudios previos, ser antagonistas de hogos fitopatógenos de suelo como Sclerotium sp., Verticillium sp., Phymatotrichopsis omnivora y Fusarium sp. (Martínez, 2016). Otra característica propia de estas especies es su potencial como promotores de crecimiento, el cual ha sido ampliamente reportado en la literatura científica, en la que se indica que son capaces de producir fitohormonas utilizadas por las plantas para su crecimiento. La caracterización convencional de las especies de Trichodermabasada en características morfológicas no es suficiente, debido a la plasticidad de caracteres, por lo que es importante realizar estudios moleculares para tener un diagnóstico con mayor certeza taxonómica (García, et al.,2017). Los análisis filogenéticos son una herramienta que apoya y valida los estudios moleculares, mediante la evidencia evolutiva que puede demostrar relaciones convergentes 0 divergentes entre especies. 
Por otro lado, se ha demostrado que Trichoderma $\mathrm{sp}$ es capaz de solubilizar fosfatos, micronutrientes y minerales tales como hierro (Fe), manganeso (Mn) y magnesio (Mg) que juegan un importante papel en el crecimiento de las plantas (Cubillos, 2009).

Debido a su amplio número de especies conocidas y alta diversidad genética, Trichoderma es un género que debe ser estudiado a nivel molecular, ya que su identificación con parámetros morfológicos es actualmente considerada insuficiente (Jaklitsch, et al., 2013). Según estudios realizados, se afirma que se ha logrado distinguir alrededor de 228 especies del género Trichoderma (Azarmi, et al., 2011), sin embargo, nuevas especies continúan siendo identificadas en la actualidad. Por lo antes descrito, el presente estudio se identificaron molecularmente especies nativas de Nicaragua pertenecientes al género Trichoderma, con el propósito de caracterizarlas morfológica y molecularmente para conocer algunas de las especies de este género que estén presentes en el país, además de realizar estudios filogenéticos de las especies identificadas.

\section{METODOLOGÍA}

\section{Aislamiento de especies de Trichoderma}

Los aislados de Trichoderma fueron extraídos de diferentes zonas de nuestro país. De las cuales 37 muestras de suelo proveniente de diferentes fuentes: terrenos agrícolas, suelos de bosques, plátano, tomate, pastos etc. se recolectaron en fincas Agrícolas de los municipios de El viejo, Tonalá, Chichigalpa y Posoltega ubicados en el Departamento de Chinandega al occidente de Nicaragua, con una extensión de $687 \mathrm{~km}^{2}$, entre las coordenadas $12^{\circ} 37^{\prime}$ de latitud norte y $87^{\circ}$ $07^{\prime}$ de longitud oeste, a una altitud de $46 \mathrm{msnm}$. Asimismo, fueron incluidas 13 muestras de suelo previamente recolectadas, una muestra de suelo perteneciente al Laboratorio de Hongos Entomopatógenos de la Universidad Nacional Autónoma de Nicaragua, León (UNAN-León), ubicado en la ciudad de León, carretera la ceiba 1 1/2 al este, 12 muestras de suelo de la Universidad Nacional de Ingeniería (UNI), provenientes de Terrabona, La libertad-Chontales y Managua para un total de 50 muestras estudiadas.

\section{Método de muestreo}

El muestreo se realizó mediante el método sistemático no probabilístico según lo recomendado por Otzen y Manterola (2017), el cual consistió en un diseño en forma de zig-zag, trazando líneas cruzadas caminando unos 25 a 30 pasos desde cada punto seleccionado de muestreo. En cada parcela se muestrearon cinco puntos, tomando 150 gramos de suelo de los primeros $15 \mathrm{~cm}$ de profundidad. Las cinco submuestras tomadas de cada parcela se homogenizaron y de ellas se tomó la muestra representativa de cada parcela de 150 gramos. Estas muestras se colocaron en bolsas plásticas y posteriormente en bolsa de papel craft, para protegerlas de los rayos UV, las cuales fueron etiquetadas para su debida identificación (Covacevich, 2017). 


\section{Aislamiento de microrganismos}

Cincuenta muestras de suelos fueron procesadas para aislar Trichoderma spp, inoculándose tres platos por cada muestra de suelo. Las muestras de suelo se procesaron mediante la metodología de Sandoval y Stefanova (2000), con el empleo de diluciones decimales seriadas. Se pesó $1 \mathrm{~g}$ de suelo y se preparó una suspensión en $9 \mathrm{~mL}$ de agua destilada estéril para preparar una suspensión madre, de la cual se realizaron 4 diluciones decimales seriadas sucesivas hasta el valor $10^{-4}$ de cada dilución (entre $10^{-1}$ y $10^{-4}$ ) se tomó $0,1 \mathrm{~mL}$ para realizar la siembra en placas Petri conteniendo medio de cultivo Agua-Agar marca Fisher (AA), más Amoxicilina(MK), utilizando $500 \mathrm{mg}$ en un litro de medio de cultivo utilizado y se sembraron tres réplicas por dilución por muestra de suelo.

Las placas se incubaron a $25^{\circ} \mathrm{C}$ por siete días realizándose observaciones diarias, a los siete días se tomó, de las placas con mayor crecimiento, una porción superficial de esporas que presentaban características similares a Trichoderma spp., se preparó una suspensión de estas y se realizó la siembra en placas con medio Papa Dextrosa Agar (PDA). A partir del crecimiento fungoso sobre este medio se tomó de cada placa, una porción de medio conteniendo micelio y se sembró en otra placa Petri hasta obtener una cepa pura.

\section{Identificación morfológica}

Las colonias puras se pasaron a tubos de ensayos que contenían PDA para su preservación a $8^{\circ} \mathrm{C}$. La identificación morfológica de las especies de Trichoderma aisladas se realizó según los criterios de Barnett y Hunter (1998). Para ello, se prepararon microcultivos de cada muestra y se realizaron observaciones microscópicas con lente objetivo 100X, para un aumento total de 1000 y así apreciar con calidad las características morfológicas de micelio, conidióforos, fiálides y conidias.

\section{Identificación Molecular de los Aislados}

La identificación molecular de los aislados se realizó con base en la amplificación de las regiones ITS1- 5.8S-ITS2 del ADNr, se utilizaron los cebadores ITS1 (5 TCC GTA GGT GAA CCT GCG G 3) (White, et al., 1990). El servicio de secuenciación de ADN se realizó en el Centro de Biología Molecular (CBM) de la Universidad Centroamericana (UCA), ubicada en Managua, Nicaragua. Una vez obtenidas las secuencias, se corrigieron utilizando el software Bioedit v7.0.9 (CastilloReyes, et al., 2015)., posteriormente se obtuvieron del GenBank del National Center for Biotechnology Information (NCBI) secuencias de las regiones estudiadas de hongos relacionadas taxonómicamente con los microorganismos, usando la herramienta BLAST (Basic Local Aligment Search Tool) para comparar secuencias altamente similares (Castillo-Reyes, et al., 2015). Se realizó un alineamiento múltiple con cinco secuencias elegidas para cada secuencia en estudio, utilizando la aplicación Clustal W Multiple alignment versión 1.4 incluida en el menú Accesory Application del programa Bioedit v7.0.9 y se utilizaron los parámetros por defecto. El árbol filogenético se construyó usando el programa MEGA 7.0.14 usando el método Neighbor-Joining Tree, evaluando la confiabilidad haciendo bootstrapping de 1000 repeticiones (Castillo-Reyes, et al., 2015; Tamura, et al., 2011; Álvarez, et al., 2013). 
El árbol filogenético se construyó usando el programa MEGA 7.0.14 usando el método NeighborJoining Tree, evaluando la confiabilidad haciendo bootstrapping de 1000 repeticiones (CastilloReyes, et al., 2015; Tamura, et al., 2011; Álvarez, et al., 2013).

\section{RESULTADOS Y DISCUSIÓN}

\section{Aislamiento de Trichoderma}

De las 50 muestras de suelo procesadas se logró aislar al hongo Trichoderma sp de 17 muestras proveniente de diversos rubros agrícolas (11c,14,13,5,10,12,9, 11a,3121G-21,8, CIXD-07, MIXD08,2,4, F2TG-11,1; Tabla 1). De los 17 aislados de Trichoderma obtenidos, cinco de ellos fueron encontrados asociados al cultivo del plátano $(11 \mathrm{c}, 3,2,4,1)$, lo que coincide con lo reportado por otros autores como Samuelian, (2016) y Pineda, et al. (2019), quienes también encontraron diferentes especies de Trichoderma en suelos agrícolas asociados al cultivo del plátano. En el caso de tomate, dos aislados fueron recuperados de tomate triturado $(14,121 \mathrm{G}-21)$ y uno de suelo asociado al cultivo del tomate(5), en este último caso, la asociación entre Trichoderma y el cultivo del tomate ha sido ampliamente documentada, y se ha indicado que los exudados radiculares de este cultivo proveen un ambiente rico en carbono y energía que crean un ambiente favorables para el desarrollo de Trichoderma en la rizósfera de este y otros cultivos (Fernández, et al., 2017 ;Haichar, et al., 2014).

En suelos asociados a bosques se logró aislar en tres ocasiones (13, 10, CIXD-07) a Trichoderma $\mathrm{sp}$, este comportamiento era esperado debido a la actividad que ejerce este organismo, sobre los ciclos biogeoquimicos, particularmente en el ciclo del carbón de los hongos asociados al suelo (Almaraz; et al., 2012). De igual manera se sabe que este tipo de hongo puede vivir en la rizósfera de las plantas forestales siendo ampliamente conocida su habilidad de competir con otros microorganismos por espacio y nutrientes. En el caso de bosques de pino, Martínez (1999) aisló una amplia variedad de hongos de suelo, siendo las poblaciones de Trichoderma spp., el género más abundante y dominante en su estudio.

De las muestras tomadas de suelos destinados a pastizales $(8,9)$, se pudo aislar el hongo Trichoderma en dos ocasiones, esto ya ha sido reportado previamente y se ha encontrado conviviendo a este hongo con este tipo de plantas sin causar ningún daño, sino actuando como organismo benéfico (Talavera \& Téllez, 2007). Finalmente, se logró aislar en una ocasión al hongo Trichoderma spp, de los cultivos de guayaba (11a), sandía (12) y suelos no agrícolas (MIXD-08) (Tabla 1). 
Tabla 1. Distribución geográfica y rubros muestreados de suelos agrícolas. Destacándose número y lugar donde fue posible la obtención de aislados de Trichoderma.

\begin{tabular}{|c|c|c|c|}
\hline Departamento & Municipios & $\begin{array}{c}\text { Rubros/ } \\
\text { muestreados }\end{array}$ & $\begin{array}{l}\text { Número y } \\
\text { códigos de } \\
\text { aislados } \\
\text { obtenidos }\end{array}$ \\
\hline \multicolumn{4}{|l|}{ Chinandega } \\
\hline & Chichigalpa & Guayaba & $\begin{array}{c}1 \\
(11 a)\end{array}$ \\
\hline & Tonalá & $\begin{array}{l}\text { Plátano } \\
\text { Bosque } \\
\text { Sandía }\end{array}$ & $\begin{array}{c}6 \\
(1,2,3,11 \mathrm{c}) \\
(13) \\
(12)\end{array}$ \\
\hline & Posoltega & Plátano & $\begin{array}{c}1 \\
(4)\end{array}$ \\
\hline León & León & Tomate & $\begin{array}{c}1 \\
(5)\end{array}$ \\
\hline Matagalpa & Terrabona & $\begin{array}{l}\text { Tomate } \\
\text { Triturado } \\
\text { Bosque } \\
\text { Tomate } \\
\text { Pastizales }\end{array}$ & $\begin{array}{c}6 \\
(\text { F2TG-11) } \\
(10) \\
(121 G-21,14) \\
(8,9)\end{array}$ \\
\hline Chontales & La Libertad & $\begin{array}{l}\text { Suelos no } \\
\text { agrícolas }\end{array}$ & $\begin{array}{c}1 \\
(\mathrm{MIXD}-08)\end{array}$ \\
\hline Managua & Ticuantepe & $\begin{array}{l}\text { Reserva Natural } \\
\text { El Chocoyero }\end{array}$ & $\begin{array}{c}1 \\
\text { (CIXD-07) }\end{array}$ \\
\hline Total & & & 17 \\
\hline
\end{tabular}




\section{Identificación Morfológica de los aislados de Trichoderma}

De las 50 muestras extraídas de diferentes Zonas de Nicaragua se encontraron 17 aislados con características morfológicas similares a las del género de Trichoderma sp. Los cuales fueron estudiadas al microscopio presentando las siguientes Características:

Los aislados 5,13,14, 10, 11a; (Tabla 3) extraídos de diferentes sustratos como son tomate triturado, suelos de bosque, guayaba y tomate, al ser observadas al microscopio sus estructuras morfológicas se pudo comprobar que mostraron similares características a las descritas por Samuels, et al. (1999), consistiendo en conidióforos con ramificaciones escasas y en pares, fiálides rectos y Conidias subglobosas con unas verrugas levemente perceptibles (Tabla 2). Otros aislados fueron (12, 9; Tabla 3) provenientes del cultivo de sandía y pastizales, esta especie al ser observada al microscopio se pudo comprobar que las estructuras morfológicas relacionadas a conidióforos, fiálides y conidias era muy similares a Trichoderma. (Masaquiza_Chango, 2019; López, 2012).

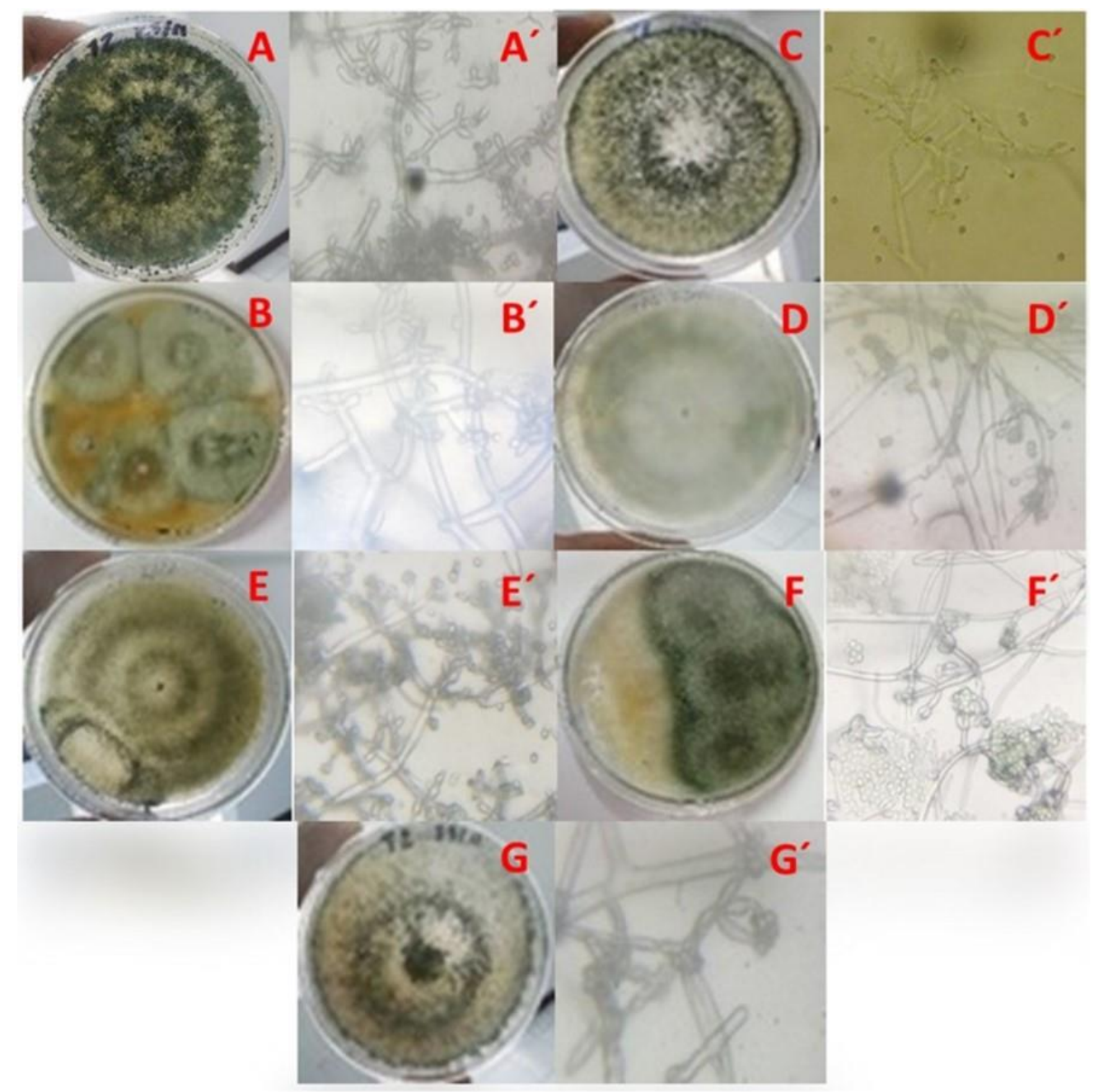

Figura 1. se observa la morfología de hongos del género Trichoderma, aislados e identificados de suelos agrícolas, donde se muestra la estructura morfológica, forma, color y crecimiento de las El Higo Revista Científica / Volumen 11. No. 01, pp. 26-42/ junio 2021 
siete especies identificadas. A, A': T. breve 2,4, F2TG-11; B, B': T. asperellum 3,5,10, 11a,13,14; C,C': T. asperelloide 12,9; D,D': T. songyi 121G-21,8; E,E': T.virens CIXD-07, MIXD-08; $\mathrm{F}, \mathrm{F}^{\prime}:$ T, viride 11c; G, $\mathrm{G}^{\prime}:$ T.harzianum 1. (Tabla 3)

En el caso de los aislados 2,4,F2TG-11; (Tabla 3) fue extraído en tres ocasiones, dos veces del cultivo del plátano y una ocasión de tomate triturado al ser observado al microscopio, las estructuras morfológicas de esta especie referidas a conidióforo, fiálides y conidias fueron congruentes con las reportadas previamente por Chen y Zhuang (2017), quienes describieron conidióforos simétricos usualmente con ramas laterales cortas, fiálides en pares o en grupos de tres y conidias color verde sin verrugas visibles. Los aislados CIXD-07, MIXD-08; (Tabla 3) fueron extraídos dos veces, una vez de suelos asociados a bosque y la otra ocasión de suelo no agrícola, morfológicamente su conidióforo se caracteriza por tener una base estéril y producir ramas cerca del ápice del mismo, sus fiálides se agrupan de 2 a 5 verticilios en ramificaciones terminales y sus conidias son elipsoidales con pequeñas verrugas en su superficie, casi imperceptible color verde oscuro. Estas características coinciden con la descripción que hicieron de este género Gams y Bissett (1998).

En el caso de los aislados 8,121G-21; (Tabla 3) se encontraron en dos ocasiones, inicialmente de tomate triturado y luego de suelos asociados a pastizales. Al observarse en el microscopio presentaba conidióforos de forma verticiliar, con fiálides angostas y rectas. Las conidias tenían coloración amarillenta a verdosas sin verrugas visibles en la superficie, estas características coinciden con las descritas por otros investigadores Park, et al., (2014). Finalmente, 11c; (Tabla 3) fue aislado en una ocasión de suelos asociados a cultivo de plátano, las observaciones de sus estructuras morfológicas realizadas a nivel de microscopio coinciden con lo descrito por Gams y Bissett (1998) para esta especie (Figura I y Tabla 2).

Tabla 2. Caracterización morfológica de especies de Trichoderma sp. aisladas de diversas fuentes (Cubillos,2009; Cruz, 2015; Park,2014; Samuels, 1999).

\begin{tabular}{|c|c|c|c|}
\hline $\begin{array}{l}\text { Especie de } \\
\text { Trichoderma }\end{array}$ & Conidióforo & Fiálides & Conidias \\
\hline $\begin{array}{l}\text { Trichoderma } \\
\text { harzianum }\end{array}$ & $\begin{array}{l}\text { Tiende a tener una forma } \\
\text { verticiliada, con una } \\
\text { estructura piramidal. }\end{array}$ & $\begin{array}{l}\text { Poseen forma entre } \\
\text { ampuliforme a } \\
\text { lageniforme, usualmente } \\
\text { 3-4 fíales verticiliados y } \\
\text { ocasionalmente en } \\
\text { pares. }\end{array}$ & $\begin{array}{l}\text { Forma subglobosa a } \\
\text { ovoide, con una } \\
\text { coloración subhialina } \\
\text { a verde pálido }\end{array}$ \\
\hline $\begin{array}{l}\text { Trichoderma } \\
\text { viride }\end{array}$ & $\begin{array}{l}\text { Usualmente no muy } \\
\text { ramificada, las ramas a } \\
\text { menudo en pares o con } \\
\text { tres verticillios } \\
\text { aparentemente flexibles. }\end{array}$ & $\begin{array}{l}\text { Frecuentemente se } \\
\text { presentan en pares o } \\
\text { grupos de tres, son } \\
\text { angosto y lageniformes }\end{array}$ & $\begin{array}{l}\text { Forma globosa a } \\
\text { elipsoidal, } \\
\text { usualmente con } \\
\text { verrugas evidentes, } \\
\text { coloración } \\
\text { verdeazulado a } \\
\text { verde oscuro. }\end{array}$ \\
\hline
\end{tabular}


María D. Sánchez M.; Luis F. Moreno M.; Leandro A. Páramo A.

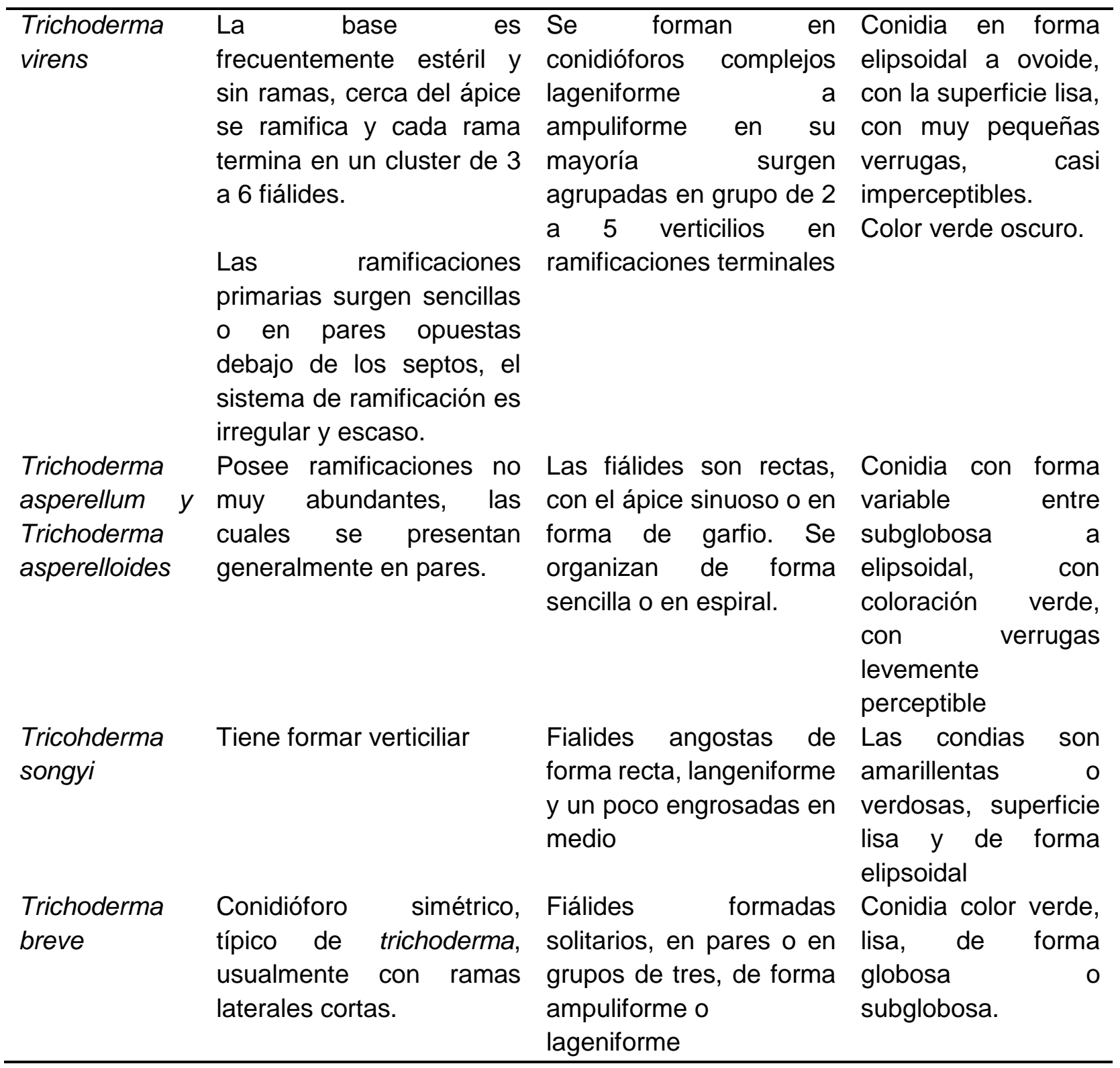

\section{Identificación Molecular de Trichoderma}

Los 17 aislados (presuntos Trichodermas, según la tabla 1) fueron enviados a secuenciar y fueron procesadas las secuencias recibidas para la elaboración del árbol filogenético construido (Una parte del cual es presentado en la Figura 2). Las secuencias resaltadas en el árbol corresponden a los microorganismos en proceso de identificación y las restantes corresponden a secuencias obtenidas del Genbank como parte del procesamiento filogenético. Se pudo determinar mediante el análisis filogenético hecho a la Figura 2, que fue posible identificar y corroborar que todos los 17 aislados pertenecen al género Trichoderma, como se habían identificado morfológicamente y descrito en la tabla 2 y figura 1 . Seis aislados de esos 17 aislados, fueron identificados como 
pertenecientes a la especie asperellum y están presentes en diversos cultivos establecidos en la región con diferentes tipos de suelos, según se estableció en la tabla 1.

Identificar este número de aislados de esta especie es muy importante, debido al reconocido potencial como agente de control biológico de enfermedades de plantas que posee, además por la diversidad de suelos y cultivos a partir de los cuales fueron aislados. Infante, et al., (2013), trabajando en condiciones in vitro, encontraron que $T$. asperellum mostró un alto nivel de control sobre Rhizoctonia solani que es un hongo de suelo que causa serios daños a una amplia variedad de cultivos entre, los cuales se encuentran los cultivos mencionados en la tabla 1. Otros estudios, desarrollados a nivel de campo, demostraron nuevamente excelentes niveles de control de esta especie sobre organismos fitopatógenos como Pyricularia grisea, Fusarium spp., $P$. pachyrhizi y Cercospora kikuchii, organismos asociados a cultivos de alta importancia agrícola como arroz, frijol y soya, lo que viene a corroborar la importancia de esta especie, dada su utilidad y potencial como agente de control biológico de enfermedades de plantas (Cruz, et al., 2015). (Figura 2 y Tabla 3). Finalmente, Duarte, et al., (2018) (trabajando también in vitro) indicaron que Trichoderma asperellum posee una alta capacidad inhibitoria en contra de hongos fitopatógenos del género Fusarium sp.

Entre los aislados identificados, además Trichoderma asperellum (previamente discutido), fue posible identificar 2 Trichoderma asperelloides. Estos fueron encontrados en cultivos muy diferentes como sandia y pastizales, en suelos y regiones tan diferentes como occidente y Matagalpa. Otros aislados identificados fueron, 1 Trichoderma viride (Solo fue aislado en occidente a partir del cultivo del plátano), 2 Trichoderma virens (ambos fueron aislados a partir de suelos no agrícolas y en regiones tan disimiles como Managua y Chontales) , 2 Trichoderma songyi (solo fue aislado en cultivos diferentes en la región de Terrabona, Matagalpa), 3 Trichoderma breve (Solo fue aislada en occidente a partir de cultivos diferentes) y 1 Trichoderma harzianum (aislada en occidente a partir del cultivo del plátano).

Diversos autores han reportado que Trichoderma virens produce una enzima denominada $\beta-1,6$ glucanasa que degrada la pared celular de hongos fitopatógenos y ha sido demostrado que dicha enzima disminuye la capacidad de Pythium ultimum de infectar plantas, lo cual fue confirmado a través de bioensayos con diferentes especies vegetales (Larralde-Corona, et al., 2008; Martínez, et al., 2013). Por otro lado, se ha indicado que la especie Trichoderma harzianum, es capaz de producir una enzima del tipo de las proteasas que degrada las enzimas que utiliza $B$. cinerea para degradar los componentes de la pared celular de las plantas (Harman, 2000) inhibiendo de esta forma su capacidad de infectarlas. De igual manera, esta especie es capaz de ejercer control sobre Rhizoctonia solani en follaje del cultivo del arroz reduciendo la incidencia de esta enfermedad en aproximadamente un 30\% (Rodríguez, 1999). Trichoderma viride por otro lado produce una enzima $\quad \beta$-glucosidasa y mediante ella degrada la fitotoxina que produce Rhizoctonia solani en las raíces de las plantas (Howel, 2003), esta especie ha mostrado un alto potencial como controlador biológico, ya que se ha demostrado que es capaz de disminuir la incidencia y severidad de patógenos que atacan el follaje de plantas, tal es el caso de Moniliophthora roreri causante de la moniliasis en cacao (Verde, 2007). 


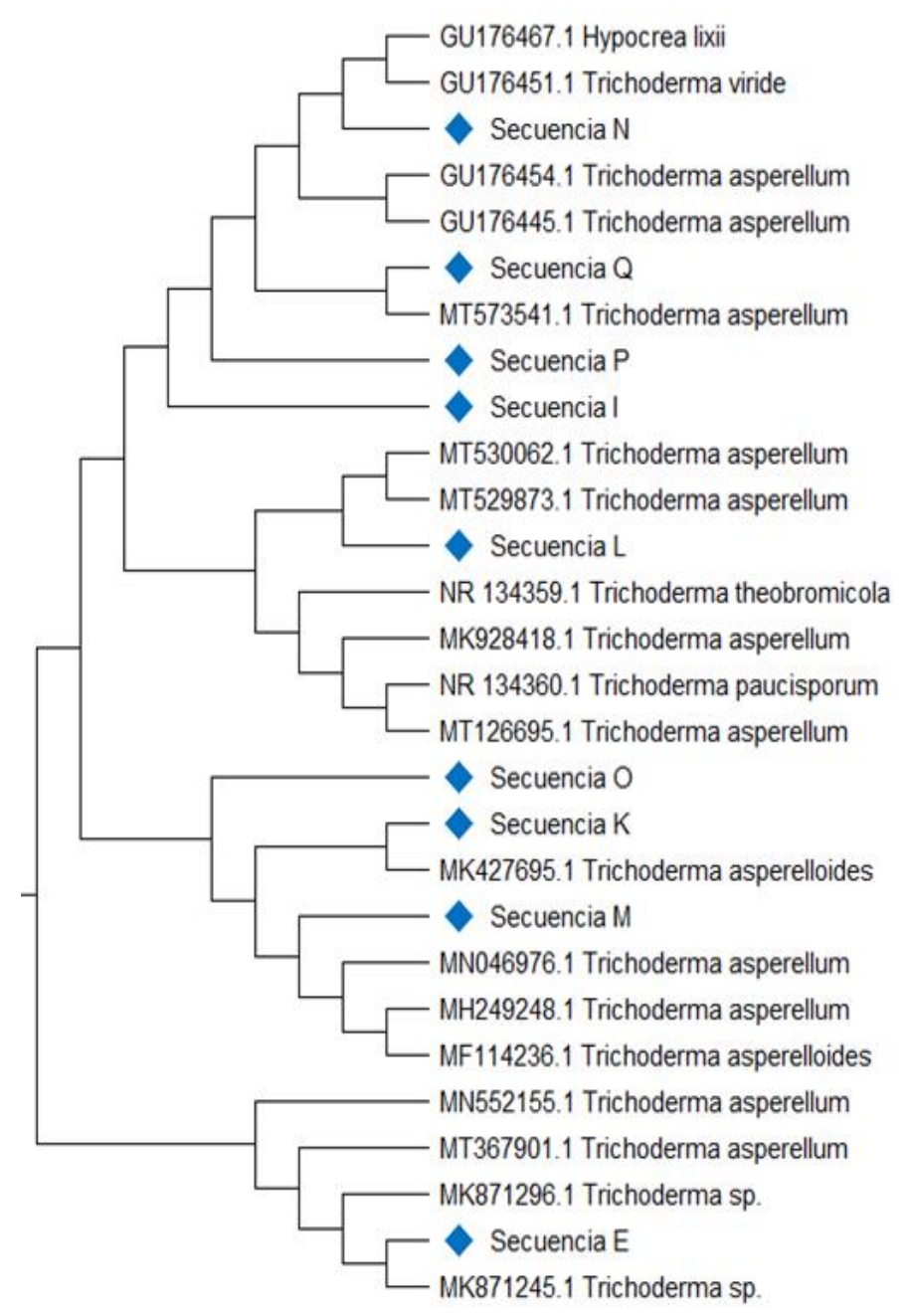

Figura 2. Árbol filogenético de diferentes especies de Trichoderma identificadas en el diagnóstico molecular.

Hasta este momento, existe una limitada información sobre la relación del hongo Trichoderma songyi, con variables agrícolas o ambientales. Sin embargo, Park, et al., (2014) identificó esta especie asociada al hongo comestible de considerable valor económico como es Tricholoma matsutake en Pino. Aunque no hay estudios que indiquen su efecto sobre este hongo, estudios previos demuestran que el género Trichoderma ha exhibido una correlación positiva cuando es encontrado en asociación con el hongo Tricholoma matsutake (Vaario, et al., 2011). Por lo que se ha sugerido que es de vital importancia desarrollar proyectos de investigación que involucren esta especie de Trichoderma (Park et al., 2014). Finalmente, el aislado correspondiente a Trichoderma breve que fue identificado en tres localidades es una especie con una muy limitada información. Pero dada la utilidad de este género para la agricultura, ambiente y farmacología entre otras áreas de la ciencia, debería retomarse esta especie para futuros estudios (Chen \& Zhuang, 2017) (Tabla 3y Figura 2). 
Tabla 3. Identificación de hongos Trichoderma mediante análisis filogenético en correspondencia con el árbol filogenético de la figura II. En la tabla se muestra el código de la secuencia y el código del microorganismo, además de la muestra de donde se aisló, puntuación e identidad máxima con el vecino cercano y la identidad final de los hongos.

\begin{tabular}{|c|c|c|c|c|c|c|c|}
\hline $\begin{array}{l}\text { Código de } \\
\text { Secuencia }\end{array}$ & $\begin{array}{c}\text { Código del } \\
\text { Microorga } \\
\text { nismo }\end{array}$ & $\begin{array}{l}\text { Aislado/ } \\
\text { Cultivo }\end{array}$ & $\begin{array}{c}\text { Procedencia } \\
\text { de la } \\
\text { muestra }\end{array}$ & $\begin{array}{c}\text { Vecino más } \\
\text { cercano } \\
\text { (Nro. de } \\
\text { acceso) }\end{array}$ & $\begin{array}{l}\text { Max } \\
\text { punt. }\end{array}$ & $\begin{array}{l}\text { Ident. } \\
\text { Max }\end{array}$ & Identidad final \\
\hline $\mathbf{N}$ & $11 \mathrm{c}$ & Plátano & Tonalá & $\begin{array}{l}\text { Trichoderma } \\
\text { viride } \\
\text { (GU176451.1 }\end{array}$ & 843 & 100 & Trichoderma viride \\
\hline $\mathbf{Q}$ & 14 & $\begin{array}{l}\text { Tomate } \\
\text { triturado }\end{array}$ & Terrabona & $\begin{array}{l}\text { Trichoderma } \\
\text { asperellum } \\
\text { (MT573541.1 }\end{array}$ & 843 & 100 & $\begin{array}{l}\text { Trichoderma } \\
\text { asperellum }\end{array}$ \\
\hline $\mathbf{P}$ & 13 & Bosque & Tonalá & $\begin{array}{l}\text { Trichoderma } \\
\text { asperellum } \\
\text { (MT573541.1 }\end{array}$ & 843 & 100 & $\begin{array}{l}\text { Trichoderma } \\
\text { asperellum }\end{array}$ \\
\hline I & 5 & Tomate & UNAN-León & $\begin{array}{l}\text { Trichoderma } \\
\text { asperellum } \\
\text { (MT573541.1 }\end{array}$ & 843 & 100 & $\begin{array}{l}\text { Trichoderma } \\
\text { asperellum }\end{array}$ \\
\hline $\mathbf{L}$ & 10 & Bosque & Terrabona & $\begin{array}{l}\text { Trichoderma } \\
\text { asperellum } \\
\text { (MT529873.1 }\end{array}$ & 907 & 99.4 & $\begin{array}{l}\text { Trichoderma } \\
\text { asperellum }\end{array}$ \\
\hline 0 & 12 & Sandia & Tonalá & $\begin{array}{l}\text { Trichoderma } \\
\text { asperelloides } \\
\text { (MK427695.1 } \\
\text { ) }\end{array}$ & 946 & 100 & $\begin{array}{l}\text { Trichoderma } \\
\text { asperelloides }\end{array}$ \\
\hline $\mathbf{K}$ & 9 & $\begin{array}{l}\text { Pastizale } \\
\text { s }\end{array}$ & Terrabona & $\begin{array}{l}\text { Trichoderma } \\
\text { asperelloides } \\
\text { (MK427695.1 } \\
\text { ) }\end{array}$ & 946 & 99.43 & $\begin{array}{l}\text { Trichoderma } \\
\text { asperelloides }\end{array}$ \\
\hline $\mathbf{M}$ & $11 a$ & Guayaba & Chichigalpa & $\begin{array}{l}\text { Trichoderma } \\
\text { asperellum } \\
\text { (MN046976.1 } \\
\text { ) }\end{array}$ & 929 & 99.61 & $\begin{array}{l}\text { Trichoderma } \\
\text { asperellum }\end{array}$ \\
\hline $\mathbf{E}$ & 3 & Plátano & Tonalá & $\begin{array}{l}\text { Trichoderma } \\
\text { asperellum } \\
\text { (MK871245 ) }\end{array}$ & 937 & 99.8 & $\begin{array}{l}\text { Trichoderma } \\
\text { asperellum }\end{array}$ \\
\hline $\mathbf{T}$ & $121 \mathrm{G}-21$ & $\begin{array}{l}\text { Tomate } \\
\text { triturado }\end{array}$ & $\begin{array}{l}\text { Terrabona- } \\
\text { Guajiniquil }\end{array}$ & $\begin{array}{l}\text { Trichoderma } \\
\text { songyi }\end{array}$ & 870 & 99.3 & $\begin{array}{l}\text { Trichoderma } \\
\text { songyi }\end{array}$ \\
\hline
\end{tabular}


María D. Sánchez M.; Luis F. Moreno M.; Leandro A. Páramo A.

\begin{tabular}{|c|c|c|c|c|c|c|c|}
\hline & & & & $\begin{array}{l}\text { (NR159053.1 } \\
\text { ) }\end{array}$ & & & \\
\hline $\mathbf{J}$ & 8 & $\begin{array}{l}\text { Pastizale } \\
\mathrm{s}\end{array}$ & Terrabona & $\begin{array}{l}\text { Trichoderma } \\
\text { atroviride } \\
\text { (H973225.1) }\end{array}$ & 939 & 99.8 & $\begin{array}{l}\text { Trichoderma } \\
\text { songyi }\end{array}$ \\
\hline $\mathbf{S}$ & CIXD-07 & Bosque & $\begin{array}{l}\text { Reserva } \\
\text { Natural "El } \\
\text { Chocoyero }\end{array}$ & $\begin{array}{l}\text { Trichoderma } \\
\text { virens } \\
\text { (MT229169.1 } \\
\text { ) }\end{array}$ & 809 & 100 & $\begin{array}{l}\text { Trichoderma } \\
\text { virens }\end{array}$ \\
\hline $\mathbf{R}$ & MIXD-08 & $\begin{array}{l}\text { Suelos } \\
\text { no } \\
\text { Agrícolas }\end{array}$ & $\begin{array}{l}\text { Chontales-La } \\
\text { libertad }\end{array}$ & $\begin{array}{l}\text { Trichoderma } \\
\text { virens } \\
\text { (NR138428.1 }\end{array}$ & 776 & 100 & $\begin{array}{l}\text { Trichoderma } \\
\text { virens }\end{array}$ \\
\hline D & 2 & Plátano & Tonalá & $\begin{array}{l}\text { Trichoderma } \\
\text { breve } \\
\text { (NR154574.1 }\end{array}$ & 802 & 99.3 & $\begin{array}{l}\text { Trichoderma } \\
\text { breve }\end{array}$ \\
\hline $\mathbf{G}$ & 4 & Plátano & Posoltega & $\begin{array}{l}\text { Trichoderma } \\
\text { breve } \\
\text { (NR154574.1 } \\
\text { ) }\end{array}$ & 802 & 99.3 & Trichoderma breve \\
\hline $\mathbf{U}$ & F2TG-11 & $\begin{array}{l}\text { tomate } \\
\text { triturado }\end{array}$ & $\begin{array}{l}\text { Terrabona- } \\
\text { Guajiniquil }\end{array}$ & $\begin{array}{l}\text { Trichoderma } \\
\text { breve } \\
\text { (NR154574.1 }\end{array}$ & 802 & 99.3 & Trichoderma breve \\
\hline $\mathbf{C}$ & 1 & Plátano & Tonalá & $\begin{array}{l}\text { Trichoderma } \\
\text { harzianum } \\
\text { (MF108885.1 }\end{array}$ & 924 & 100 & $\begin{array}{l}\text { Trichoderma } \\
\text { harzianum }\end{array}$ \\
\hline
\end{tabular}

\section{CONCLUSIONES}

El aislamiento de 50 muestras de Suelos extraídas de diferentes zonas del país permitió obtener 17 aislados que presentaban características similares a las de Trichoderma. Las observaciones morfológicas realizadas, permitieron presumir que existían coincidencias entre lo reportado y lo observado a nivel de laboratorio, además con la realización de las pruebas moleculares, el análisis filogenético y lo publicado por otros autores, permitió concluir en que los 17 aislados pertenecían a las especies: T. asperellum 14, 13, 5,10, 3, 11a, T. asperelloides 12,9; T. breve, 2 , 4, F2TG-11; T. harzianum 1 Trichoderma virens CIXD-07, MIXD-08; T. songyi 121G-21,8; Trichoderma viride (11c) con porcentajes de identidad que oscilan de 99 a 100\%. Los aislados identificados por vía molecular, procedían de diferentes regiones del país y fueron aislados en diferentes tipos de cultivos. De gran importancia resulta el haber obtenido aislados tan efectivos en el combate de plagas como T.asperellum y una nueva puerta de investigación se abre con aislados de $T$. breve y de $T$. songyi, ya que muy poco se conoce sobre la aplicación de estas especies en la agricultura u otros campos de interés biotecnológico 


\section{REFERENCIAS}

Álvarez, C., Osorio, N., \& Montoya, M. (2013). Identificación molecular de microorganismos asociados a la rizósfera de plantas de vainilla en Colombia. Acta Biológica Colombiana, 293-305.

Almaraz-Sánchez, A., Alvarado-Rosales, D., Tlapal-Bolaños, B. \& Espinoza-Victoria, D. (2012). Identificación de hongos antagonistas a Phytophthora cinnamomi rands en bosques de encino del Arrayanal, Colima y Tecoanapa, Guerrero. Revista Chapingo. Serie Ciencias Forestales y del Ambiente 18:341-356

Azarmi, R., Hajieghrari, B. \& A. Giglou. (2011). Effect of Trichoderma isolates on tomato seedling growth response and nutrient uptake. African Journal of Biotechnology 10(31): 5850-5855.

Barnett, H. y Hunter, B. (1998). Illustrated general of imperfect fungi. 4 ed. New York: Mc Millan Publishing Company. $241 \mathrm{p}$.

Castillo-Reyes, F., Hernández-Castillo, F., gallegos-Morales, G., Flores-Olivas, A., RodríguezHerrera, R., \& Aguilar, C. (2015). Efectividad in vitro de Bacillus y polifenoles de plantas nativas de México sobre Rhizoctonia solani. Revista Mexicana de Ciencias Agrícolas, 549562.

Chen, K. \& Zhuang, W.Y. (2017) Discovery from a large-scaled survey of Trichoderma in soil of China. Scientific Reports 7: 9090.

Covacevich, F. 2017. Hongos Micorricicos arbusculares: Muestreo de suelo para determinación de actividad y diversidad de hongos micorrícicos arbusculares. En: Metodología de muestreo de suelo y ensayos a campo: Protocolos básicos comunes. D. J. Santos, M. Wilson y M. Ostinelli (Eds.) Ediciones INTA

Cruz A, Rivero D, Echevarría A, Infante D \& Martínez B. (2015). Trichoderma asperellum en el manejo de hongos fitopatógenos en los cultivos de arroz (Oryza sativa L.), frijol (Phaseolus vulgaris L.) y soya (Glycine max L.). Rev. Protección Veg.; 30(suppl.1):87p.

Cubillos-Hinojosa J., L. Mejía, y Valero. N. (2009). Trichoderma harzianum como promotor del crecimiento vegetal del maracuyá (Passiflora edulis var. flavicarpa Degener). Agron. Colomb. 27: 81-86

Duarte-Leal, Y; Pozo-Martínez, L. \& Martínez-Coca, B. (2018). Antagonismo in vitro de cepas de Trichoderma asperellum Samuels, Lieckfeldt \& Nirenbergfrente a aislados de Fusarium spp. Rev. Protección Veg., vol.33, n.1

Fernández E, Trillas MI \& Segarra G, (2017). Increased rhizospere populations of Trichoderma asperellum strain T34 caused by secretion pattern of root exudates in tomato plants inoculated with Botrytis cinerea. Plant Pathology.

Gams, W., \& Bissett, J. (1998). Morphology and identification of Trichoderma. In Harman, G.E. and Kubicek, C.P. (Eds.), Trichoderma and Gliocladium, Vol (1), Basic Biology, Taxonomy and Genetics. (pp. 3-34). London, England: Taylor and Francis Ltd. 
García H; Martínez A, Hermosa M, Monte E; Aguilar C; y González C. (2017). Caracterización morfológica y molecular de cepas nativas de Trichoderma y su potencial de biocontrol sobre Phytophthora infestans. Rev. mex. fitopatol vol.35 no.1 Texcoco

Haichar FEZ, Santaella C, Heulin T \& Achouak W, 2014. Root exudates mediated interactions belowground. Soil Biology and Biochemistry 77, 69-80.

Harman G. (2000). Myths and dogmas of control. Changes in perceptions derived from research on Trichoderma harzianum T-22. Plant Disease.; 84(4):377-393.

Howell C. (2003).Mechanisms employed by Trichoderma species in the biological control of plant diseases: The history and evolution of current concepts. Plant Diseases.; 87(1):4-10.

Infante D, Martínez B, Pereira B, Reyes Y, Herrera A. (2013). Identificación molecular y evaluación patogénica de trece aislamientos de Trichoderma spp. Frente a Rhizoctonia solani Kühn. Biotecnol Apl.; 30 (1):17-22

Jaklitsch W.M., Samuels G.J., Ismaiel A. \& Voglmayr H. (2013). Disentangling the Trichoderma viride scens complex. Persoonia 31: 112-146.

Larralde-Corona C, Santiago M, Sifuentes A, Rodríguez I, Shirai K, \& Narváez J. (2008) Control potential and polyphasic characterization of novel native Trichoderma strains against Macrophomina phaseolina isolated from sorghum and common bean. Appl Microbiol Biotechnol. 80:167-177.

López, C. L. Á. (2012). Identificación y caracterización bioquímica, morfológica y molecular de microorganismos cultivables asociados a la rizósfera y al sustrato de plantas de vainilla. (Tesis de Maestría presentada para optar al título de Magíster en Ciencias-Geomorfología y Suelos), universidad Nacional de Colombia, Colombia.

Martínez, B.; Infante, D. \& Reyes, R. Y. (2013). Trichoderma spp. y su función en el control de plagas en los cultivos. Revista de Protección Vegetal. 1(28):1-11.

Martínez-Scott, M. (2016). Evaluación de aislados nativos de Trichoderma sp para el control de hongos fitopatógenos del suelo en tomate. Revista de Ciencias Naturales y Agropecuarias. Vol.3 No.6 32-42

Martínez, A. E. (1999). Estudio ecológico de los hongos del suelo de un bosque de Pinus Taeda. Buenos Aires. Trabajo de grado (Doctorado en Ciencias Biológicas. Universidad de Buenos Aires. Facultad de Ciencias Naturales.

Masaquiza Chango, C. E. (2019). Caracterización morfológica y molecular de hongos asociados a la rizósfera de plantas de café en la isla Santa Cruz-Galápagos (Bachelor's thesis, Quito: UCE).

Otzen T, Manterola C. (2017). Técnicas de muestreo sobre una población a estudio. International Journal of Morphology. 
Park, M.S., Oh, SY. Cho, H.J., Fong J.J., Cheon WJ, \& Lim, YW. (2014). Trichoderma songyi sp. nov., a new species associated with the pine mushroom (Tricholoma matsutake). Antonie van Leeuwenhoek 106, 593-603

Pineda, M.; Pineda, D.; Labarca, J.; Ulacio, D.; Paredes, C. \& Casassa, A. M. (2019). Micobiota del suelo asociada al cultivo del plátano (Musa aab cv. Hartón) en bosque seco tropical del Sur del Lago de Maracaibo, Venezuela. Revista Udo Agrícola. 9 (1):158-165.

Rodríguez H, Nass H, Cardona R \& Alemán L. (1999). Alternativas para controlar el añublo de la vaina, causado por Rhizoctonia solani del arroz. Fitopatología Venezolana.; 12(1):18.

Samuelian, S. (2016). Potential of Trichoderma harzianum for control of banana leaf fungal pathogens when applied with a food source and an organic adjuvant. 3 Biotech. 6 . 10.1007/s13205-015-0327-0.

Sandoval I \& Stefanova M. (2000). Efectividad de biopreparados de Trichoderma spp. en el control de los hongos fitopatógenos del suelo. Boletín Técnico No. 2. Instituto de Investigaciones de Sanidad Vegetal (INISAV). La Habana, Cuba. 22 p.

Samuels, G. J., E. Lieckfeldt \& H. I. Nirenberg (1999). Trichoderma asperellum, a new species with warted conidia, and redescription of T. viride. - Sydowia 51(1): 71-88.

Talavera, A., \& Téllez, M. (2007). Hongos patógenos, especies amigables con el ambiente. Revista. Inventio 27(12):41-45. Disponible en: http://inventio.uaem.mx/index.php/inventio/arti. cle/view/167/413

Tamura, K., Peterson, D., Peterson, N., Stecher, G., Nei, M., \& Kumar, S. (2011). MEGA5: Molecular Evolutionary Genetics Analysis using Maximum Likelihood, Evolutionary Distance, and Maximum Parsimony Methods. Molecular Biology and Evolution 28, 27312739.

Vaario L-M, Fritze H, Spetz P, Heinonsalo J, Hanajı́k P, \& Pennanen T (2011) Tricholoma matsutake dominates diverse microbial communities in different forest soils. Appl Environ Microbiol 77(24):8523-8531

Verde W. (2007). Dos Hongos Antagónicos (Trichoderma harzianum y Trichoderma viride). Efecto en el control de la Moniliasis (Moniliophthora roreri Cif y Par. Evans et al.) del cacao en la Región Ucayali. Tesis. Ing. Agr. UNAS. Perú. 70 pp

White TJ, Bruns T, Lee S, Taylor JW. (1990). Amplification and direct sequencing of fungal ribosomal RNA genes for phylogenetics. En: Innis MA, Gelfand DH, Sninsky JJ, White TJ, editors. PCR Protocols: A guide to methods and applications. New York: Academic Press;. p. 315-322.

\section{AGRADECIMIENTOS}

A la vicerrectoría de la Universidad Nacional Autónoma de Nicaragua -León, por el medio de su vicerrector el PhD. Wilbert Salazar, por su apoyo económico para el desarrollo de la investigación. 
Al Programa de Investigación Estudios Nacionales y Servicios Ambientales (PIENSA-UNI), por medio de su directora la MSc. Larisa Korsak, por la colaboración en infraestructura de laboratorio y apoyo Técnico en biotecnología para que la presente investigación culminara con éxito.

\section{SEMBLANZA DE LOS AUTORES}

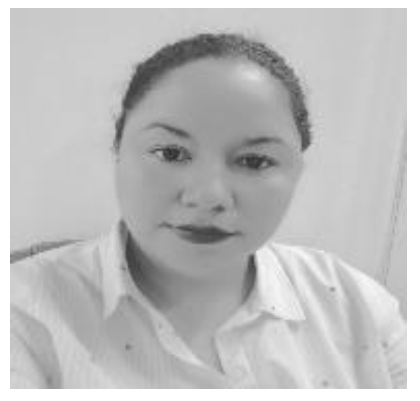

María Delfina Sánchez- Miranda: Obtuvo el grado en ingeniería en Agroecología tropical, en la Universidad Nacional Autónoma de Nicaragua - León, Nicaragua, donde actualmente es profesor adjunto 3/4 de tiempo .Participo en curso de Desarrollo rural, con enfoque en mejoramiento de vida en el país de Japón y Guatemala, Trabaja en el Departamento de Agroecología de la Escuela de Ciencias Agrarias y Veterinaria específicamente en el área de los laboratorios de Fitopatología diagnosticando enfermedades en plantas.

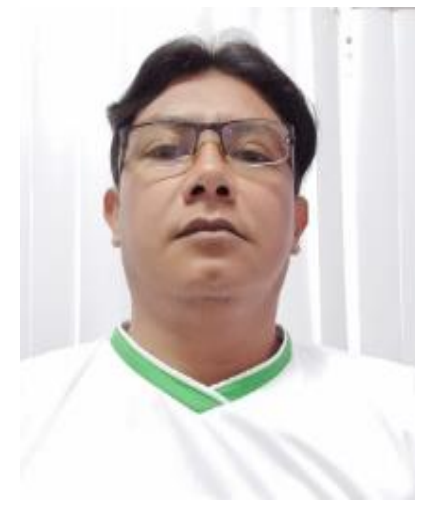

Luis Francisco Moreno Mayorga, Graduado como ingeniero en agroecología Tropical, en la Universidad Nacional Autónoma de Nicaragua, UNAN-León, diplomado en calidad inocuidad y trazabilidad de productos agrícolas en la Universidad Nacional Agraria, Maestrante en desarrollo Local Sostenible UNAN-León, experiencia en investigación, producción y transferencia de control biológico, entomopatógenos y entomófagos. Actualmente Profesor Asistente del departamento de Agroecología y director del Centro de Investigación y Reproducción de Controladores Biológicos de la Escuelas de Ciencias Agrarias y Veterinarias de UNAN-León.

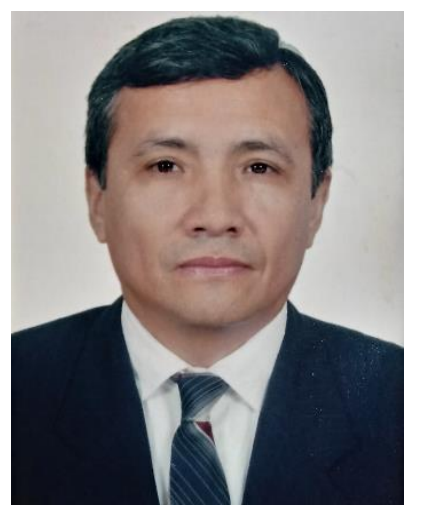

Leandro Alberto Páramo Aguilera, Graduado como Ingeniero Químico con maestría en Ingeniería Química y énfasis en procesos biotecnológicos, en el Instituto Superior Politécnico "José Antonio Echeverría", ISPJAE, de la Ciudad de la Habana, Cuba, en el año 1990. En 1997 se gradúa como Master en Microbiología y énfasis en bacteriología en la Universidad de Costa Rica, UCR. En junio del 2012, se gradúa como Doctor en Ciencias en el área de Biotecnología en el Centro de Biotecnología Genómica (CBG) del Instituto Politécnico Nacional (IPN) de México. Amplia experiencia en el desarrollo de procesos biotecnológicos (biofertilizantes, bebidas alcohólicas, fermentados lácteos, bioprospección, compostaje, desarrollo de bioprocesos varios, desde la identificación del microorganismo hasta el desarrollo del proceso biológico para la agricultura, el ambiente y la industria. etc). 